\title{
PADRÕES CLIMATOLÓgICOS ASSOCIADOS A EVENTOS DE SECA NO LESTE DO ESTADO DE SÃO PAULO
}

\author{
GOZZO, Luiz Felippe - luiz.gozzo@unesp.br \\ Universidade Estadual Paulista Julio de Mesquita Filho / UNESP \\ PALMA, Dóris Santos - dorispalma.santos@gmail.com \\ Universidade Estadual Paulista Julio de Mesquita Filho / UNESP \\ CUSTÓDIO, Maria de Souza - m.custodio@unesp.br \\ Universidade Estadual Paulista Julio de Mesquita Filho / UNESP
}

DRUMOND, Anita - anita.drumond@unifesp.br

Universidade Federal de São Paulo / UNIFESP

Submetido em: 03/09/2020

Aceito para publicação em: 13/01/2021

Publicado em: 06/04/2021

DOI: http://dx.doi.org/10.5380/abclima.v28i0.76268

\begin{abstract}
RESUMO: Os mecanismos de ocorrência de secas no Sudeste do Brasil são complexos, pois a região encontra-se na zona de transição entre os regimes atmosféricos tropical e extratropical. Este trabalho apresenta as condições climatológicas médias sazonais no início de eventos de seca meteorológica na faixa leste do estado de São Paulo no período de 1901 a 2010. Os eventos foram identificados utilizando o índice de evapotranspiraçãoprecipitação padronizado (SPEI) calculado a partir dos dados da reanálise ERA20C. Durante o verão e o outono, o início dos eventos é associado a uma teleconexão atmosférica originada por anomalias quentes do oceano na região da Indonésia. Um padrão de bloqueio no Pacífico Sul desfavorece o avanço de perturbações de médios e altos níveis em direção à América do Sul, e o transporte de umidade das regiões tropicais em direção a São Paulo é desfavorecido pelo enfraquecimento do Jato de Baixos Níveis. No inverno, as secas se iniciam associadas à intensificação da Alta Subtropical sobre a costa do Sudeste por um trem de ondas barotrópico propagando-se desde o Índico, e ao aumento da temperatura média e da evapotranspiração durante a fase negativa da Oscilação Antártica. O escoamento de grande escala associado aos eventos de seca iniciados na primavera remete ao padrão de teleconexão da La Niña. Os índices climáticos mais relacionados à série temporal do SPEI na faixa leste de São Paulo são da Oscilação Antártica (outono e inverno), da oscilação do Atlântico Tropical Sul (outono e primavera), e do El Niño-Oscilação Sul (primavera).
\end{abstract}

PALAVRAS-ChAVE: eventos de seca, SPEI, São Paulo, teleconexões.

\section{CLIMATE PATTERNS ASSOCIATED TO DROUGHT EVENTS IN EASTERN SAO PAULO}

ABSTRACT: Climate patterns leading to the occurrence of drought events in Southeastern Brazil are complex because they happen in a transition zone between extratropical and tropical regimes. This article presents the mean seasonal climatological conditions in the beginning of meteorological drought events in the eastern portion of the Sao Paulo state during the period 1901-2010. The events were identified using the Standardized Precipitation-Evapotranspiration Index (SPEI) in the 1 month timescale. During austral summer and autumn, drought events are associated with an atmospheric teleconnection from the Indonesia region. A blocking pattern in South Pacific blocks upper level perturbations in their way toward South America, and the weaker Low Level Jet reduces the moisture transport from lower latitudes toward Sao Paulo. In winter, droughts begin with the intensification of the Subtropical High by a barotropic wavetrain propagating from the Indian Ocean, and with increasing temperature and 
evapotranspiration during negative phases of the Antartic Oscillation. In spring, the large scale flow resembles the La Niña teleconnection pattern. Climatic indexes of Antarctic Oscillation, Tropical South Atlantic Oscillation and El Niño-Southern Oscillation presented moderate and statistically significant correlations with SPEI time series in this region.

KEYWORDS: drought events, SPEI, Sao Paulo, teleconnections.

\section{INTRODUÇÃO}

Evento de seca é um período de tempo durante o qual se verifica disponibilidade de água inferior à demanda em uma certa região (Redmond, 2002). Os efeitos desse déficit de água se acumulam enquanto o evento persiste, causando diferentes impactos. De acordo com a duração da seca, ela pode ser classificada como: meteorológica, agrícola, hidrológica e socioeconômica (Wilhite e Glantz, 1985). A seca meteorológica é definida como insuficiência de água em escala temporal da ordem de um mês, resultante de acumulados de chuva inferiores à média climatológica e/ou aumento da evapotranspiração potencial (Hanel et al., 2018). Conforme o evento se prolongue, pode afetar a disponibilidade de água do solo (seca agrícola - em torno de 3 meses), impactar a quantidade de água de reservatórios, lagos, rios, aquíferos (seca hidrológica - 6 a 12 meses), e influenciar na disponibilidade de produtos e serviços por falta de água (seca socioeconômica, 12 meses e além).

No Brasil, são numerosos os trabalhos que estudaram secas nas regiões Nordeste e Norte, onde se verifica marcadamente o impacto do El NiñoOscilação Sul (ENSO - Penalba e Rivera, 2016; Marengo et al., 2017; Vargas e Veiga, 2017; etc.) e/ou dos padrões de anomalia de temperatura da superfície do mar (TSM) da bacia do Atlântico (Jiménez-Muñoz et al., 2019; Ciemer et al., 2020; etc.) em tais eventos.

Já sobre os estados do Sudeste do Brasil, localizados em latitudes subtropicais, na faixa de transição de regime entre as circulações dos trópicos e dos extratrópicos, os efeitos destes padrões climáticos sobre a chuva são menos definidos. A região de transição entre anomalias positivas e negativas de chuva associadas ao ENSO, por exemplo, tende a se localizar na parte norte do estado de São Paulo (SP); logo, variações na posição desta transição podem modificar totalmente o sinal da oscilação sobre a região (Coelho et al., 2002). Além dos efeitos variáveis do ENSO, as condições de TSM do Atlântico também têm importante papel no regime de chuvas do Sudeste (Bombardi et al., 2014).

Climatologicamente, o estado de São Paulo (SP) pode ser basicamente dividido em duas regiões homogêneas de precipitação (Keller Filho et. al, 2005): região centro-oeste, com chuvas muito concentradas no período de verão e mais comumente observadas em forma de trovoadas isoladas, e região leste, adjacente ao Oceano Atlântico Sul, com chuva ligeiramente melhor distribuída ao longo do ano (embora as estações seca e chuvosa sejam ainda bem marcadas), persistentes e mais frequentemente associadas a fenômenos organizados como ciclones extratropicais e frentes frias que passam pela costa ou brisas marítimas (Silva et al., 2009; Vemado e Pereira-Filho, 2016). Considerável diferença entre a climatologia de secas para as regiões leste e centro-oeste do estado é documentado por Gozzo et al. (2019b), sugerindo que fatores climáticos distintos podem estar associados a estes eventos em cada região. 
A seca no leste de SP causa sérios problemas relacionados ao abastecimento e distribuição de água, pois é a região mais densamente povoada do estado, e inclui a capital (com mais de 20 milhões de habitantes em sua região metropolitana) e importantes reservatórios hídricos. Um recente episódio de seca muito intenso em 2014/2015 teve forte impacto sócio-econômico sobre o leste de SP, e foi atribuído em parte a uma teleconexão com a TSM da bacia do oceano Pacífico, via propagação de ondas de Rossby (Nobre et al., 2016; Coelho et al., 2016, Finke et al., 2020).

Melhorar o entendimento de condições atmosféricas e oceânicas de grande escala associadas ao surgimento dos eventos de seca no leste de SP pode auxiliar em previsões sazonais que afetam a agricultura e a disponibilidade hídrica e ajudar a avaliar e compreender efeitos das mudanças climáticas na intensidade e frequência destes eventos. Assim, este trabalho tem como objetivo apresentar padrões gerais de TSM e circulação atmosférica associados ao início de eventos de seca ocorridos entre 1901 e 2010 sobre o leste de SP. Os eventos serão identificados através do índice Standardized Precipitation Evaptranspiration Index - SPEI (Vicente-Serrano et al., 2010), que determina eventos secos e chuvosos anômalos a partir do balanço hidrológico (diferença entre a precipitação e a evapotranspiração potencial sobre uma região). A vantagem do SPEI sobre outros índices já existentes é incluir o efeito da temperatura do ar sobre as condições de seca. Isto permite levar em conta a influência da variabilidade de alta frequência da temperatura (como ondas de calor) e das mudanças climáticas (Dubreuil et al., 2019). Este índice é amplamente utilizado em análises de diferentes tipos de secas sobre todo o planeta (Drumond et al., 2019 e referências). Pereira et al. (2018) e Gozzo et al. (2019a) utilizaram o SPEI para estudos climatológicos de eventos de seca sobre SP. Porém, não investigaram os padrões atmosféricos de grande escala associados a estes eventos, sendo este o objetivo principal do presente trabalho.

O artigo está organizado como se segue: na seção 2 apresentamos os dados e as metodologias utilizadas nesta investigação; a seção 3 analisa e discute os resultados obtidos; a seção 4 traz as conclusões e sugestões para a continuidade das pesquisas.

\section{DADOS E METODOLOGIA}

\subsection{DADOS}

Para a análise das condições anômalas da circulação de grande escala no surgimento dos eventos de seca, foram utilizados dados de altura geopotencial em 700 e $200 \mathrm{hPa}$, vento horizontal em 850 e 200 hPa, TSM e radiação de onda longa emergente no topo da atmosfera (OLR) obtidos a partir da reanálise ERA20C, produzida pelo European Centre of Medium-Range Weather Forecast (ECMWF) (Poli et al., 2016), com resolução horizontal de $125 \mathrm{~km}$, no período entre 1901 e 2010. A pressão ao nível do mar (PNMM) utilizada para o cálculo do índice da Oscilação Antártica (AAO) foi da reanálise NOAA-CIRES-DOE Twentieth Century Reanalysis - 20CR, que possui melhor correlação e menores erros estatísticos do que a ERA-20C sobre a região considerada no cálculo do índice (Fogt et al., 2016). 
Como o estudo da circulação de grande escala foi realizado a partir de dados da ERA20C, para o cálculo do SPEI optou-se por utilizar as médias mensais (de temperatura do ar e precipitação acumulada) provenientes da mesma reanálise, com o objetivo de homogeneizar a origem dos dados. Os eventos de seca identificados na ERA-20C foram comparados aos obtidos com dados do Climate Research Unit (CRU - Harris et al., 2017), a partir da precipitação medida por estações meteorológicas em resolução horizontal de $0.5^{\circ}$. A validação da climatologia foi realizada com o intuito de comparar a ERA20C com as observações do CRU e identificar se esta reanálise é adequada para o estudo dos eventos de seca na região.

\subsection{METODOLOGIA}

\subsubsection{CÁlCULO DO SPEI}

Os eventos de seca foram identificados na escala temporal de um mês (seca meteorológica - SPEI-1) na área do leste de SP (daqui em diante referida como ESP e destacada na Figura 1).

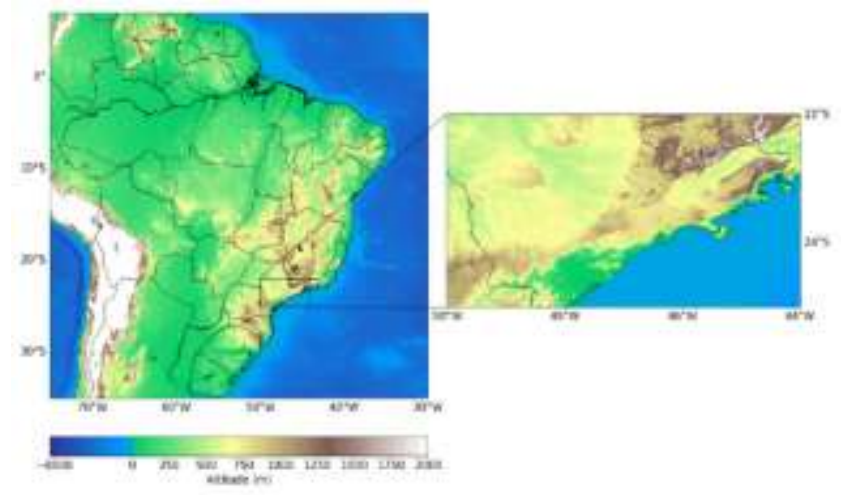

Figura 1 - Domínio da área de estudo (leste de SP - ESP), com altitude em m (fonte: NCEI-NOAA).

Para o cálculo do SPEI, primeiramente foram calculadas as médias espaciais de temperatura do ar e precipitação acumulada sobre a ESP, para cada mês, de janeiro de 1901 a dezembro de 2010. A taxa de evapotranspiração potencial (ETP) média mensal na área foi estimada pelo método de Thorntwaite (1948), utilizando a temperatura média mensal e a quantidade de horas de fotoperíodo em determinada latitude. Ainda que seja bastante simples, por considerar apenas a temperatura do ar como variável independente, o método é um dos que apresentam melhor desempenho sobre SP (Camargo e Sentelhas, 1997), sendo por isso escolhido para este estudo.

A diferença entre a precipitação de um mês $i$ e a ETP média deste mês fornece o balanço hidrológico $D_{i}=P_{i}-E T P_{i}$. A série temporal de $D_{i}$ foi ajustada a uma distribuição de probabilidade teórica (log-logística) e normalizada. O índice SPEI para cada mês foi obtido pela comparação entre $D$ no mês $i$ e a probabilidade da ocorrência de tal valor na climatologia daquele mês, dada pela distribuição normalizada. São determinados, assim, meses com condições extremas mensais do balanço hídrico. Detalhes sobre a metodologia de cálculo do SPEI podem ser encontrados em Vicente-Serrano et al. (2010) e, 
especificamente para a reanálise utilizada neste trabalho, em Gozzo et al. (2019a).

Os eventos de seca na ESP foram identificados segundo o critério de McKee et al. (1993): eles se iniciam no mês em que o SPEI-1 apresenta valor negativo e terminam no mês em que o índice volta a ser positivo, desde que durante este espaço de tempo (mesmo que em apenas 1 mês) seja atingido valor igual ou inferior a -1.0. Também seguindo McKee et al. (1993), eventos que apresentaram pelo menos um mês com mínimo inferior a -1.5 foram caracterizados como severos, e eventos com mínimo valor de SPEI-1 inferior a 2.0 definiram eventos de seca extrema (Tabela 1).

Tabela 1 - Limiares de valores do índice SPEI para definição das categorias de intensidade dos eventos de seca e probabilidade de ocorrência de cada intervalo (Fonte: McKee et al., 1993)

\begin{tabular}{ccc}
\hline Valor do SPEI & Categoria & Probabilidade \\
\hline-1.00 a -1.49 & seca moderada & $9.2 \%$ \\
\hline-1.50 a -1.99 & seca severa & $4.4 \%$ \\
\hline$\leq-2.00$ & seca extrema & $2.3 \%$ \\
\hline
\end{tabular}

\subsubsection{ANÁLISE DE COMPOSIÇÕES}

Após a identificação das secas na ESP, foram construídas composições a fim de determinar os padrões meteorológicos e de TSM associados ao início dos eventos. Para este cálculo, utilizou-se os meses de início de todos os 153 eventos detectados na ERA20C, divididos nas estações meteorológicas de verão (DJF), outono (MAM), inverno (JJA) e primavera (SON). As composições de anomalias de TSM, vento zonal e meridional em 850 e $200 \mathrm{hPa}$, altura geopotencial em $700 \mathrm{hPa}$ e função de corrente $(\Psi)$ em $200 \mathrm{hPa}$ foram calculadas a partir da média do período (1901-2010) e com indicação da significância estatística superior a $95 \%$ segundo o teste t-Student. Os campos de $\psi$, que representam a componente rotacional do campo de vento horizontal, indicam anomalia de circulação anticiclônica (ciclônica) em regiões negativas (positivas) no hemisfério sul, e foram calculados para latitudes maiores que $15^{\circ}$, em ambos os hemisférios. Regiões de anomalia anticiclônica desta variável, bem como regiões de anomalia positiva de altura geopotencial em $700 \mathrm{hPa}$, indicam predomínio de movimentos verticais descendentes na coluna atmosférica, e alta pressão em superfície.

\subsection{3. ÍNDICES CLIMÁTICOS}

Os índices climáticos do Oceanic Niño Index (ONI), Tropical South Atlantic (TSA) e South Atlantic Subtropical Dipole Index (SASDI) foram calculados a partir da TSM da ERA20C. Anomalias positivas/negativas de TSM sobre o Pacífico Tropical na área entre $170^{\circ}-120^{\circ} \mathrm{W}$ e $5^{\circ} \mathrm{N}-5^{\circ} \mathrm{S}$ (Niño 3.4) configuram o índice ONI positivo/negativo (Trenberth, 1997), assim como anomalias positivas/negativas no leste do Atlântico $\left(30^{\circ} \mathrm{W}-10^{\circ} \mathrm{E}, 20^{\circ} \mathrm{S}-0^{\circ}\right)$ determinam o TSA positivo/negativo (Enfield et al., 1999). Já o SASDI é calculado como a diferença entre as anomalias do oceano Atlântico subtropical $\left(20^{\circ} \mathrm{W}-0^{\circ}, 25^{\circ} \mathrm{S}-15^{\circ} \mathrm{S}\right)$ e extratropical $\left(30^{\circ} \mathrm{W}-10^{\circ} \mathrm{W}, 40^{\circ} \mathrm{S}-30^{\circ} \mathrm{S}\right)$ - o índice é 
positivo/negativo quando há anomalia negativa/positiva de TSM na região mais ao norte e positiva/negativa na área mais ao sul (Morioka et al., 2011)

O índice da Oscilação Antártica (AAO) foi calculado a partir da anomalia mensal de pressão ao nível do mar da reanálise 20CR em relação à média 19812010, padronizada e projetada na primeira componente principal do campo de pressão do hemisfério sul (Baldwin, 2001).

\section{RESULTADOS E DISCUSSÕES}

Foram identificados 153 eventos de seca sobre a ESP na reanálise ERA20C, no período entre 1901 e 2010. Antes da análise de composições, foi realizada uma validação da climatologia, comparando-a com as observações da análise CRU durante este período. Os dois conjuntos de dados apresentaram apenas um evento de diferença, e características médias como duração, severidade e intensidade dos eventos foram simulados sem diferenças significativas. A validação da ERA20C mostra semelhança em relação ao CRU, assim como também ocorre em outras escalas do SPEI, como 3, 12 e 48 meses (Gozzo et al., 2019a), qualificando esta reanálise como um conjunto de dados apropriado para o estudo da climatologia de secas na região ESP.

\subsection{COMPOSIÇÕES}

\subsubsection{VERÃO}

O verão é a estação chuvosa no estado de São Paulo. O sistema de monção está em sua fase ativa (Zhou e Lau, 1998; Liebmann e Mechoso, 2011; Carvalho e Cavalcanti, 2016), com a circulação na baixa troposfera transportando grandes quantidades de umidade desde as latitudes tropicais (oceano Atlântico tropical e região amazônica) para as regiões Sudeste e Sul do país. Esta umidade, em média, chega até a ESP através do jato de baixos níveis (JBN) e também de uma circulação associada ao Anticiclone Subtropical do Atlântico Sul (ASAS), que traz umidade do oceano Atlântico, entre $20^{\circ} \mathrm{S}$ e $10^{\circ} \mathrm{S}$, para a região (Marengo et al., 2004; Gozzo et al., 2017). Durante esta época do ano, o ASAS está enfraquecido e deslocado para leste na bacia (Sun et al., 2017), favorecendo a predominância de ventos úmidos de nordeste próximo ao sudeste do Brasil. Em altos níveis, ocorrem as circulações da Alta da Bolívia e do cavado do Nordeste (Chen et al., 1999); a difluência entre estes dois escoamentos, em altos níveis favorece a formação de bandas convectivas que podem se estender desde o norte do país até o sudeste e sul, e eventualmente caracterizar episódios de Zona de Convergência do Atlântico Sul (Silva et al., 2019).

Para o período 1901-2010 da reanálise ERA20C, foram identificados 40 eventos de seca na ESP com início durante o verão. Destes, 17 registraram intensidade severa e 3 atingiram categoria extrema (iniciados em jan/1906, dez/1935 e dez/1972), segundo os limiares apresentados na Tabela 1.

A composição de anomalias de função de corrente geostrófica em 200 hPa e altura geopotencial em $700 \mathrm{hPa}$ (Fig. 2a) para os 40 eventos de seca de verão apresentam dois centros anticiclônicos barotrópicos em $120^{\circ} \mathrm{W}$ e $0^{\circ}$, em torno de $45^{\circ} \mathrm{S}$. Eles são semelhantes aos anticiclones observados no trem de 
ondas em resposta à anomalia positiva de TSM na Indonésia por Coelho et al. (2016), cuja posição aproximada está indicada por uma curva preta na Fig. 2a. Um aquecimento anômalo ao norte/nordeste da Austrália também ocorre na composição de anomalias de TSM (Fig. 2b).

Apesar do campo de TSM apresentar significativa anomalia negativa sobre o Pacífico equatorial, as anomalias de altos níveis não se assemelham ao padrão Pacífico-América do Sul (PSA), sugerindo que os eventos de seca desta estação não estão tipicamente ligados à fase fria do El Niño-Oscilação Sul (La Niña). Isto é corroborado por baixa correlação entre o índice do Niño 3.4 e as secas na ESP durante o verão (seção 3.3). Já no Pacífico subtropical, a presença de duas anomalias em torno de $120^{\circ} \mathrm{W}$ (Fig. 2a) indicam um padrão de bloqueio, que dificulta a passagem de transientes que poderiam perturbar as condições de tempo no Sudeste do Brasil.

A circulação de bloqueio no Pacífico também aparece bem configurada e significativa no escoamento em altos níveis (Fig 2d). Neste campo, também é possível observar duas anomalias anticiclônicas significativas: uma centrada no norte da Argentina $\left(60^{\circ} \mathrm{W}-30^{\circ} \mathrm{S}\right)$, sugerindo um enfraquecimento do Jato Subtropical em todo o sul do Brasil e em SP, e outra sobre o Nordeste $\left(40^{\circ} \mathrm{W}\right.$ $\left.10^{\circ} \mathrm{S}\right)$, indicando enfraquecimento do Cavado do Nordeste, escoamento desfavorável para formação de zonas de convergência no Brasil Central. Além disso, uma região de difluência se estabelece sobre o Atlântico ( $30^{\circ} \mathrm{W}-25^{\circ} \mathrm{S}$ ). Em $850 \mathrm{hPa}$, o estado de SP, bem como todo o Sudeste e Centro-Oeste do país apresentam anomalias de vento de sudeste, indicando enfraquecimento do fluxo de umidade tropical em direção aos subtrópicos. Até mesmo o transporte de umidade do Atlântico Tropical em direção ao continente está reduzido nestes episódios (Fig. 2c). A desintensificação do ASAS (anomalia ciclônica em $30^{\circ} \mathrm{W}$ $25^{\circ} \mathrm{S}$ ), associada à difluência em altos níveis já mencionada também diminui o fluxo de umidade dos ventos de nordeste em direção a SP entre $20^{\circ} \mathrm{S}$ e $10^{\circ} \mathrm{S}$. Com pouca disponibilidade de vapor d'água, a nebulosidade e a chuva diminuem significativamente sobre a ESP, que apresenta anomalias de radiação de onda longa emergente (OLR) acima de $+10 \mathrm{~W} \mathrm{~m}^{-2}$, e sobre o Rio de Janeiro (RJ) e centro-sul de Minas Gerais (MG - com anomalias positivas de OLR ainda mais intensas). Já na região Nordeste, principalmente entre Bahia e Piauí, ocorrem anomalias negativas de OLR, indicando nebulosidade acima da média. Este padrão pode estar associado à ocorrência de zonas de convergência em baixos níveis nesta região. Movimentos ascendentes ao longo desta zona de convergência podem estar sendo compensados por movimentos subsidentes ao sul, sobre o Sudeste, reforçando o tempo firme entre SP, RJ e sul de MG. 

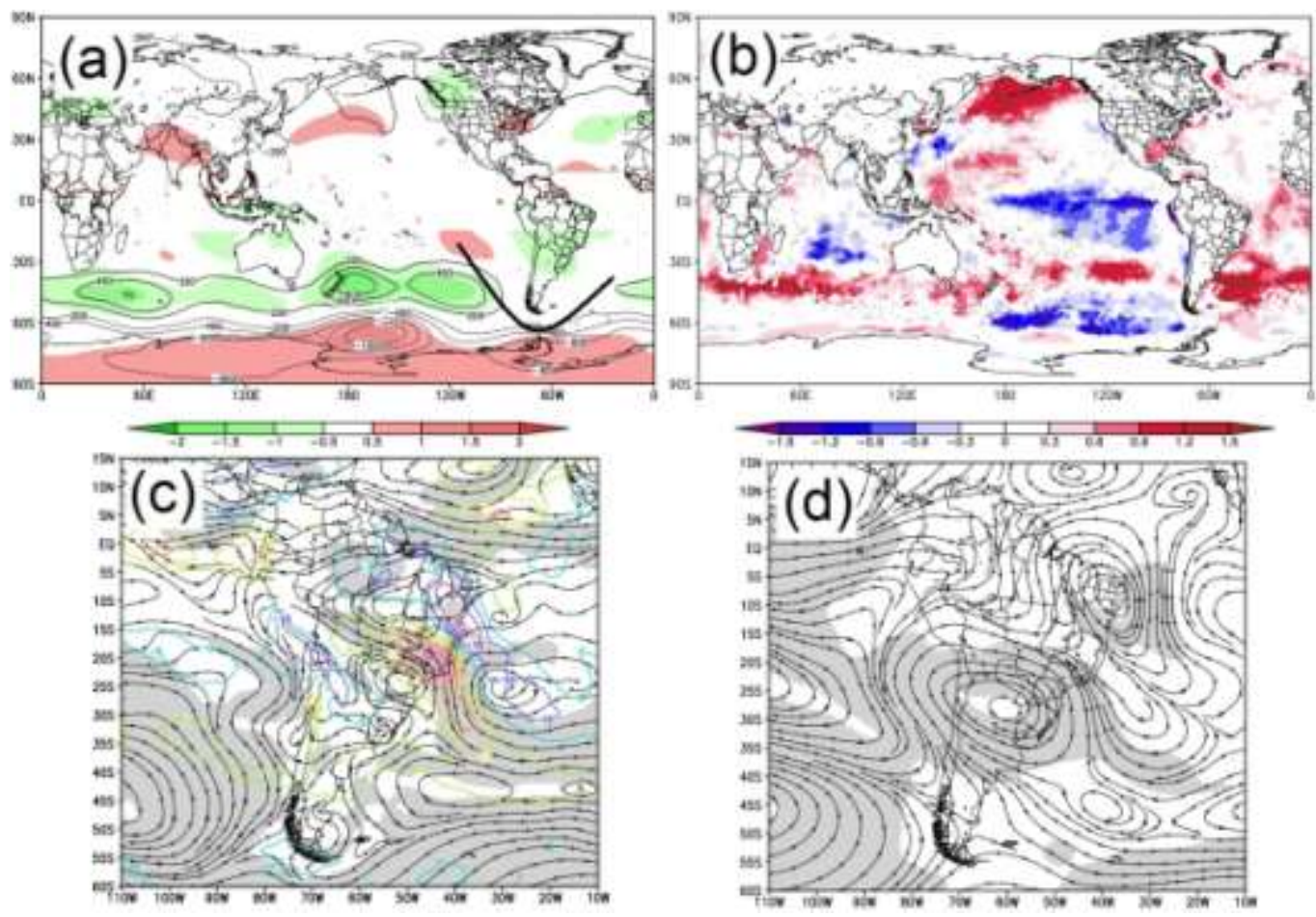

Figura 2 - Composições de anomalias de (a) função de corrente em $200 \mathrm{hPa}$ (sombreado, $10^{7} \mathrm{~m}^{2} \mathrm{~s}^{-1}$ ) e altura geopotencial em $700 \mathrm{hPa}$ (linhas, m), (b) TSM $\left({ }^{\circ} \mathrm{C}\right.$ ), (c) circulação em $850 \mathrm{hPa}$ (linhas de corrente) e OLR (contornos, W m²), e (d) circulação em $200 \mathrm{hPa}$ (linhas de corrente) associadas aos eventos de seca iniciados durante o verão. As anomalias foram calculadas em relação à média 1901-2010. Em (a) e (b) apenas anomalias com significância estatística superior a $95 \%$ foram representadas; em (c) e (d) as regiões com significância superior a 95\% foram demarcadas em cinza.

\subsubsection{OUTONO}

O outono marca a fase de transição entre a estação chuvosa do verão e o período seco do inverno, para SP. Climatologicamente, neste período do ano o ASAS começa a se intensificar e deslocar-se para oeste em direção ao continente sul-americano; o vento em baixos níveis ainda advecta umidade desde a borda norte do ASAS em direção ao leste de SP, embora com menor intensidade do que no verão (Gozzo et al., 2017). Ciclones extratropicais tornam-se mais numerosos e intensos na costa leste do Sudeste do Brasil, devido ao maior contraste entre oceano e atmosfera (Reboita et al., 2010a,b). A ZCIT atinge a sua posição mais ao sul do Equador, marcando a estação chuvosa no norte do Nordeste do Brasil.

Foram identificados 41 eventos de seca no ESP iniciados durante o outono, entre 1901 e 2010. Com relação à intensidade, 18 eventos foram severos e 2 foram extremos (início em mar/1915 e abr/1918).

O escoamento em $850 \mathrm{hPa}$ observado nas composições novamente evidencia uma desconfiguração do transporte de umidade do Brasil central em direção às regiões Sul e Sudeste, e um enfraquecimento do ASAS diminuindo o aporte de umidade desde o flanco norte da Alta em direção a SP (Fig 3c). Assim como no verão, a TSM segue mais aquecida na região da Indonésia, associada a 
uma teleconexão que pode estar intensificando o padrão de bloqueio barotrópico em torno de $110^{\circ} \mathrm{W}$ (Fig. 3a e 3d), provocando condições atmosféricas menos perturbadas sobre a América do Sul (AS) subtropical. Isto promove uma anomalia anticiclônica significativa centrada no sul do Mato Grosso do Sul, em $55^{\circ} \mathrm{W}-25^{\circ} \mathrm{S}$ (Fig. 3c), enfraquecendo $\mathrm{O}$ Jato Subtropical sobre SP e desfavorecendo a ciclogênese. Com o ambiente mais estável, as anomalias de OLR tornam-se positivas sobre grande parte do Sudeste (Fig. 3c).

Ainda no campo de anomalia de OLR, nota-se forte anomalia negativa no norte do Nordeste do Brasil, que sugere um deslocamento anômalo para sul da Zona de Convergência Intertropical (ZCIT), favorecido pelo padrão anômalo de TSM (mais quente no Atlântico ao sul do Equador, e mais frio ao norte, na costa do leste da África - Fig. 3b). Este deslocamento resulta em chuvas acima da média sobre o Nordeste do Brasil (Moura e Shukla, 1981; Uvo et al., 1998; Saravanan e Chang, 2000), e pode favorecer subsidência de compensação sobre o Brasil central, levando a menores acumulados de precipitação sobre SP.

\subsubsection{INVERNO}

Durante o inverno, SP atravessa a estação seca, com o sistema de monção da América do Sul inativo e a ASAS mais intensificada e atuando sobre o sudeste do Brasil (Sun et al., 2017). A disponibilidade de umidade no estado é baixa, e os movimentos verticais ascendentes são desfavorecidos. Na ERA20C entre 1901 e 2010, foram identificados no inverno 32 eventos de seca, com 12 eventos severos e 3 extremos (iniciados em jul/1914, jun/1936, jul/1967).

O campo de composição de anomalias de função de corrente geostrófica em $200 \mathrm{hPa}$ e altura geopotencial em $700 \mathrm{hPa}$ mostra um trem de ondas barotrópico bem configurado ao longo da latitude de $45^{\circ} \mathrm{S}$, estendendo-se desde o oceano Índico até o leste da América do Sul, passando por toda a bacia do Pacífico. Ao se aproximar do continente sul-americano, este padrão se curva em direção às baixas latitudes, posicionando um centro anticiclônico anômalo sobre SP (Fig. 4a). O padrão de ondas é semelhante ao segundo modo de variabilidade (EOF) do vento em $200 \mathrm{hPa}$, fortemente correlacionado com anomalias de precipitação no Uruguai observados por Cazes-Boezio et al. (2003). Apesar do campo de TSM novamente mostrar anomalias frias das águas na região do Niño 3.4 durante os eventos de seca, Cazes-Boezio et al. (2003) discutem que o padrão de circulação anômala em altos níveis não é significativamente correlacionado com a TSM sobre o Pacífico Tropical, e que surge entre os meses de maio e julho por conta de mudanças na frequência e amplitude de regimes de circulação intrassazonais nos extratrópicos. Esta dissociação com o Niño 3.4 é reforçada pela correlação fraca e não significativa entre SPEI1 e ONI para a ESP (seção 3.3). 

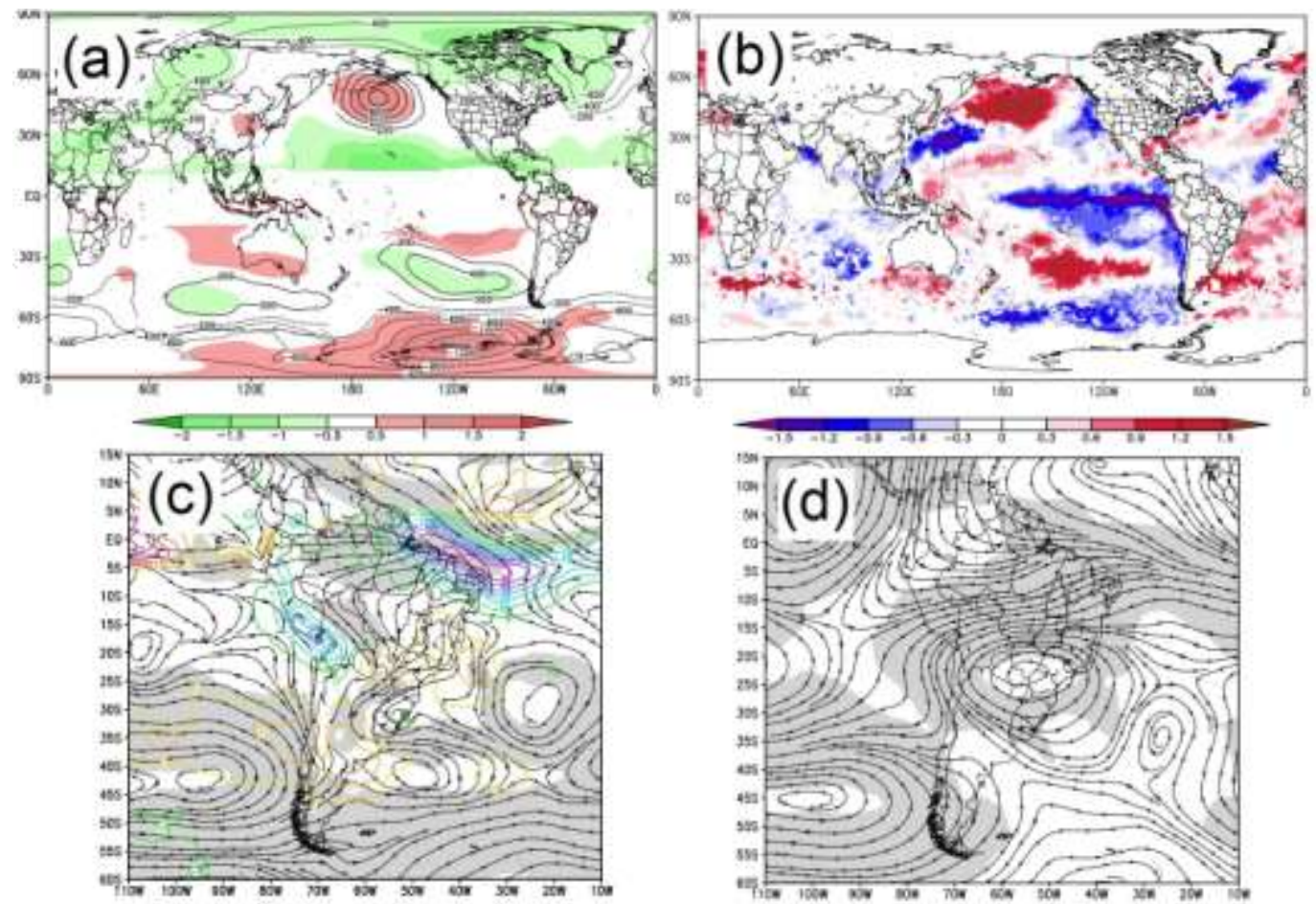

Figura 3 - Como na Fig. 2, para os eventos de seca iniciados no outono.
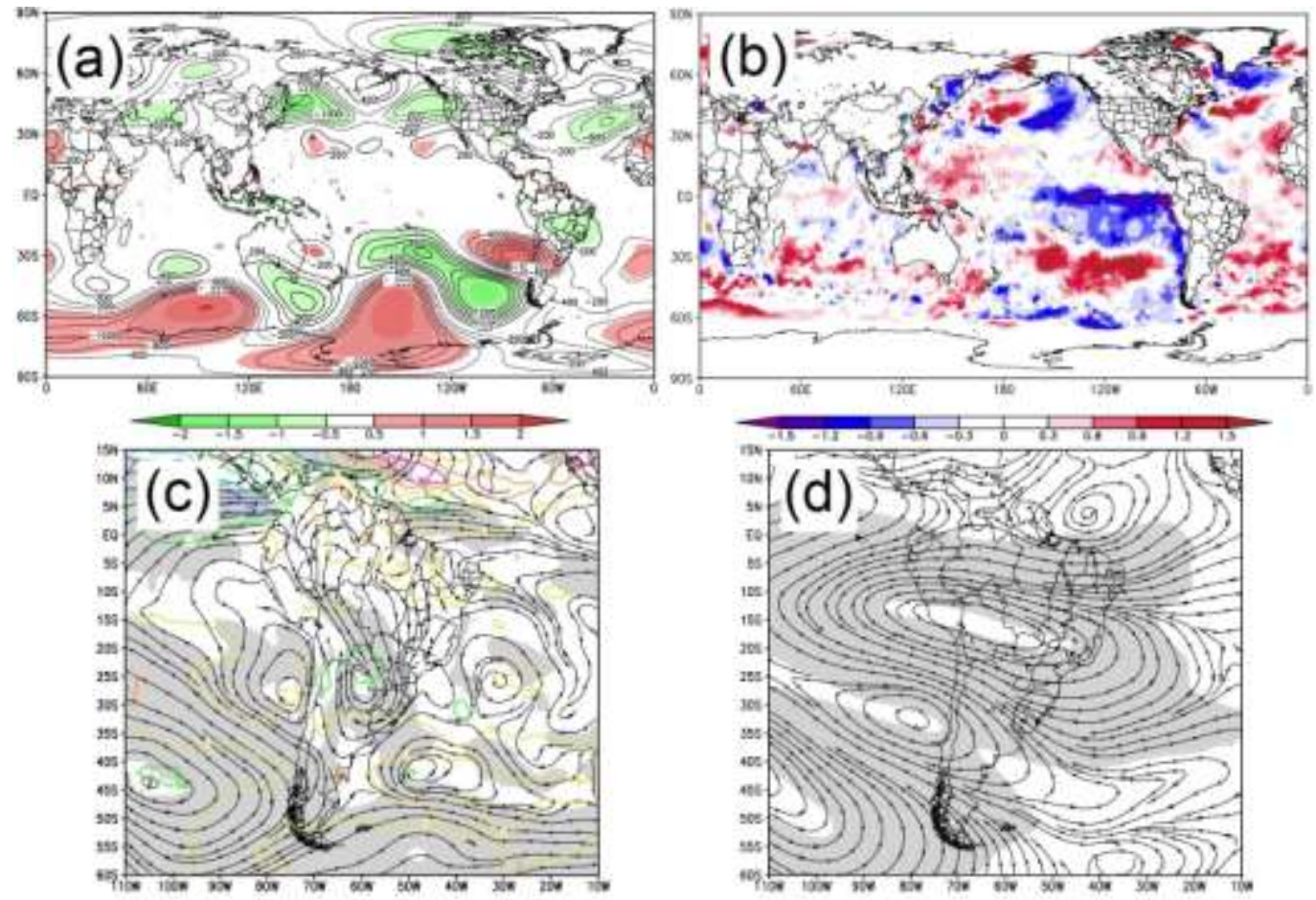

Figura 4 - Como na figura 2, para os eventos de seca iniciados no inverno. 
Os eventos secos no inverno estão associados a uma extensa e significativa circulação anticiclônica em altos níveis sobre a AS (Fig. 4d). Em conjunto com um ciclone anômalo centrado em $80^{\circ} \mathrm{W}-30^{\circ} \mathrm{S}$, esta circulação provoca uma intensificação do jato de altos níveis, o que favorece abaixamento anômalo de pressão sobre o norte da Argentina. Esta baixa anômala pode ser vista no campo de anomalia de vento em $850 \mathrm{hPa}$ (Fig. 4c), direcionando eventuais fluxos de ar mais úmido das latitudes mais altas para uma posição a oeste do estado de São Paulo. Ainda neste campo, a anomalia anticiclônica sobre o Atlântico (centrada em $30^{\circ} \mathrm{W}-25^{\circ} \mathrm{S}$, e possivelmente associada à anomalia anticiclônica em altos níveis sobre o Sudeste do Brasil) indica um fortalecimento do ASAS, inibindo movimentos ascendentes.

\subsubsection{PRIMAVERA}

Na primavera ocorre a transição da estação seca para a estação chuvosa em SP. O sistema de monção da América do Sul entra em sua fase ativa, e a circulação do JBN passa a trazer mais umidade das baixas latitudes em direção ao sul/sudeste novamente. O ASAS climatologicamente perde força e se afasta do continente, facilitando movimentos ascendentes em SP.

Nos meses de primavera do período 1901-2010, surgiram, segundo a ERA20C, 39 eventos de seca. Deste total 13 foram severos, e 3 apresentaram intensidade extrema (estes foram iniciados em nov/1910, out/1922, nov/1990).
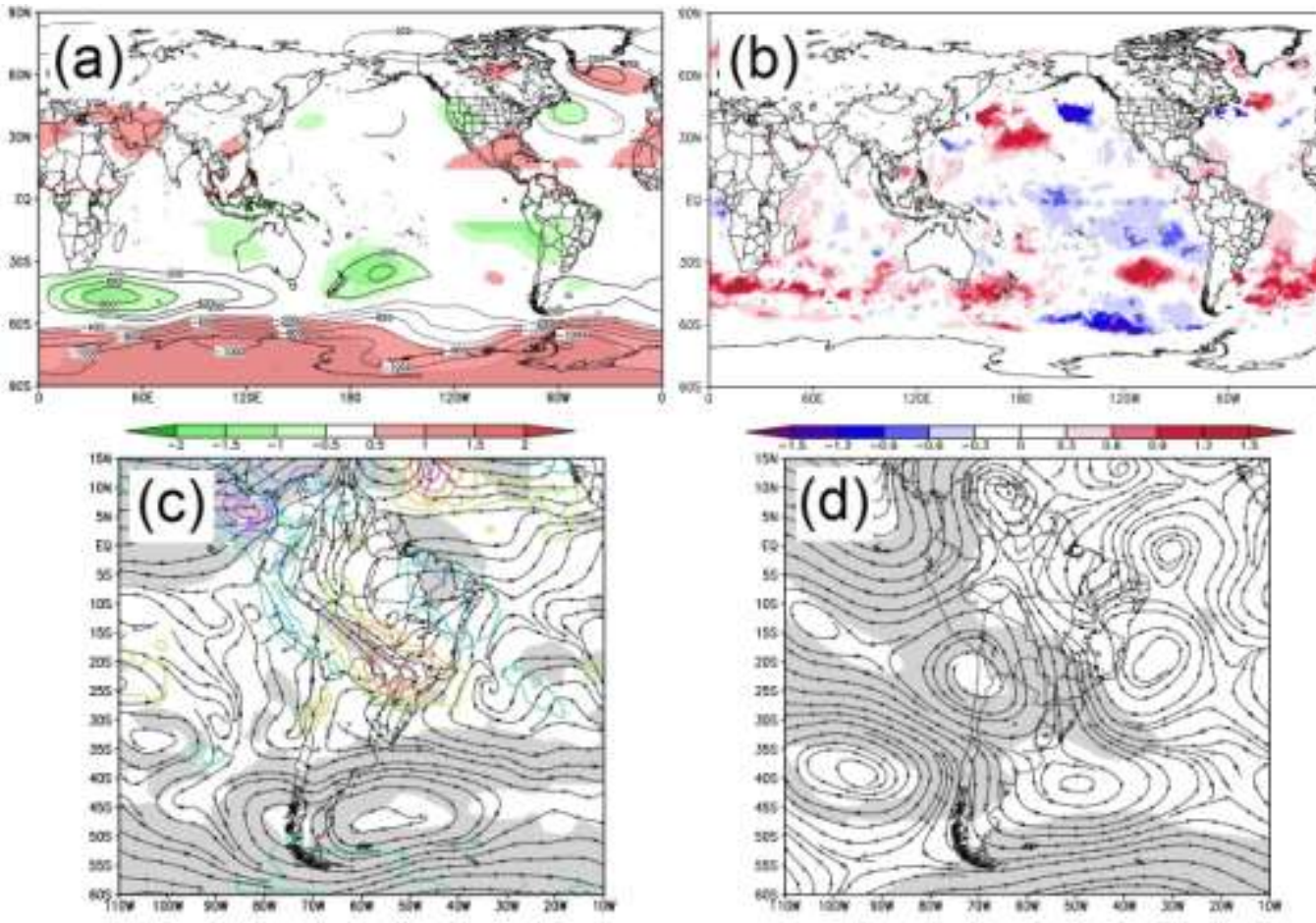

Figura 5 - Como na figura 2, para os eventos de seca iniciados na primavera

Nos eventos de seca iniciados durante a primavera, há sinal de um trem de ondas com centros em torno de $35^{\circ} \mathrm{S}$, nas longitudes de $170^{\circ} \mathrm{W}, 100^{\circ} \mathrm{W}$ e $55^{\circ} \mathrm{W}$ (Fig. 5a) que se assemelha ao padrão PSA. Como este padrão é forçado pelas anomalias de TSM no Pacífico Tropical (Mo e Paegle, 2001) a Fig. 5a 
sugere uma possível relação entre o ENSO e o início dos eventos de seca na primavera sobre a ESP. No campo de anomalia de vento em 200 (Fig. 5d), observa-se dois centros anticiclônicos anômalos, um sobre o norte do Chile $\left(70^{\circ} \mathrm{W}-20^{\circ} \mathrm{S}\right)$ e outro sobre o Atlântico $\left(50^{\circ} \mathrm{W}-40^{\circ} \mathrm{S}\right)$, com uma anomalia ciclônica a nordeste $\left(30^{\circ} \mathrm{W}-20^{\circ} \mathrm{S}\right)$, novamente remetendo a um padrão de onda associado ao PSA durante eventos de LN (Mo e Paegle, 2001; Pereira et al., 2017). O par de anomalias ciclone-anticiclone ao longo de $20-25^{\circ}$ de latitude sul enfraquece 0 jato subtropical sobre SP (desloca-o para norte de sua posição climatológica) e intensifica a advecção de vorticidade anticiclônica sobre a região sul do país e SP, inibindo movimentos ascendentes (Grimm, 2004).

O escoamento em altos níveis estabelecido tem efeitos evidentes sobre o vento em baixos níveis reforçando as condições para seca na ESP (Fig. 5c). Novamente, observa-se o JBN com uma significativa anomalia de ventos de sudeste, transportando portanto menos umidade para latitudes mais baixas durante os eventos secos de primavera. A desintensificação (intensificação) do JBN durante episódios de LN (El Niño) e seu impacto na precipitação no sudeste da AS é um efeito documentado na literatura (Grimm et al., 1998; Zhou e Lau, 2001; Lau e Zhou, 2003), associado à desintensificação (intensificação) do jato de altos níveis subtropical sobre o Sul/Sudeste do Brasil. O padrão geral de anomalia ciclônica a leste da AS e anticiclônica a oeste resulta em um escoamento anômalo de sul em baixos níveis sobre o Brasil central, minimizando o transporte de umidade do Atlântico Tropical e do flanco norte do ASAS em direção ao Sul/Sudeste; este padrão é comum nos meses de primavera de anos de LN (Grimm, 2004).

Os campos médios indicam condições atmosféricas anômalas tipicamente associadas à LN, corroborando trabalhos anteriores, porém o campo de TSM do Niño 3.4 mostra as mais fracas anomalias no período da primavera (Fig. 5b). Isto pode estar associado a uma resposta tardia da atmosfera às condições de LN bem estabelecida no inverno. Ou pode ser um artifício das composições, pela inclusão de eventos de seca ocorridos em primaveras do ano imediatamente anterior ao estabelecimento do evento de LN, quando as TSMs tendem a apresentar anomalia positiva (Penalba e Rivera, 2016; Anderson et al., 2017). Mesmo assim, o sinal de resfriamento do Pacífico Tropical está presente durante os eventos de seca, com anomalias entre $-0,3$ e $-0,9^{\circ} \mathrm{C}$, significativas ao nível de $95 \%$.

Embora o campo médio de TSM do Pacífico não indique uma anomalia fria tão intensa, parece provável que condições de LN sejam uma forçante importante para disparar eventos de seca na primavera: a maior parte dos 39 eventos identificados na reanálise foi iniciada nas primaveras de anos de LN (43\%). Eventos iniciados em primaveras de anos neutros e de El Niño foram, respectivamente, $31 \%$ e $26 \%$ do total.

\section{2. ÍNDICES CLIMÁTICOS}

As análises de composições da seção anterior sugerem que o balanço hídrico em superfície sobre a ESP é modulada por padrões de TSM em escala climatológica, como o ENSO e os padrões do Atlântico. Para avaliar essa hipótese calculamos a correlação entre o índice SPEI-1 na ESP e quatro índices climáticos, apresentados na Tabela 2. Durante o verão, as correlações são 
praticamente nulas e não significativas, indicando que os padrões climáticos analisados aqui têm fraca relação simultânea com eventos extremos (secos e chuvosos) na ESP. Para o outono, moderada correlação negativa com a TSA (o que já era esperado pelo campo de anomalias de OLR sobre o NE do BR) e positiva com a AAO, ambas significativas ao nível de $99 \%$. Também moderada e altamente significativa é a correlação positiva entre o SPEI-1 e a AAO durante o inverno. Na primavera, o ONI aparece com correlação moderada e significativa ao nível de $99 \%$ com o SPEI-1; moderada, mas um pouco menos significativa, é a correlação com a TSA.

Tabela 2 - Correlação de Pearson entre a série temporal do SPEI-1, suavizada com uma média móvel de cinco anos, e índices climáticos. $\left(^{*}\right)$ e $(* *)$ indicam correlações estatisticamente significativas ao nível de $95 \% \quad(p<0.05)$ e $99 \% \quad(p<0.01)$, respectivamente, segundo teste t-Student. Em negrito, as maiores e mais significativas correlações.

\begin{tabular}{lcccc}
\cline { 2 - 5 } & ONI & AAO & SASDI & TSA \\
\hline Summer & 0.1 & 0.1 & -0.1 & 0.0 \\
\hline Autumn & $0.2(*)$ & $\mathbf{0 . 5}(* *)$ & $0.2(*)$ & $\mathbf{- 0 . 4 ( * * )}$ \\
\hline Winter & -0.2 & $\mathbf{0 . 6 ( * * )}$ & 0.0 & 0.0 \\
\hline Spring & $\mathbf{0 . 3 ( * * )}$ & 0.1 & 0.0 & $\mathbf{- 0 . 3 ( * )}$ \\
\hline
\end{tabular}

A correlação mais forte e significativa entre o SPEI1 da ESP e o ONI na primavera concorda com resultados de Grimm et al. (2000) onde os efeitos mais fortes do ENSO no sudeste da AS são sentidos nesta estação. O trabalho mostra que durante o verão as anomalias negativas de chuva enfraquecem e se deslocam para oeste em direção à Argentina; elas voltam no final do verão para o extremo sul do país, com forte variabilidade durante o outono. Embora o seu trabalho seja focado no Uruguai, Cazes-Boezio et al. (2003) também documenta maior influência do padrão de teleconexão associado ao ENSO durante a primavera, e o quase desaparecimento deste padrão sobre o sudeste da AS durante o verão.

Já em relação aos índices do Atlântico, a correlação da TSA e o SPEI1 no outono indica que o aquecimento do Atlântico Tropical deve provocar uma condição atmosférica análoga à descrita por Bombardi et al (2014): enfraquecimento da ASAS e menor aporte de umidade para a região Sudeste, além de anomalias positivas dos ventos equatoriais de leste em $200 \mathrm{hPa}$ e desenvolvimento de uma circulação anticiclônica em altos níveis próxima a SP (Figs. 3c e 3d). Na primavera, embora a correlação entre o SPEI1 e o TSA apareça com força e significância semelhantes ao outono, esse padrão não aparece claro na anomalia de TSM (5b). Planejam-se estudos posteriores para detalhar a relação da TSM do Atlântico com as chuvas de SP, mas destaca-se aqui que Pampuch et al. (2016) encontrou a mesma relação de seca em grande parte de SP com a área da TSA. Porém, as outras regiões do Atlântico apresentavam anomalias diferentes das vistas aqui - o que pode ser devido à diferente metodologia de escolha dos eventos de seca, identificados a partir de dados diários de precipitação. Em relação ao SASDI, a única estação com correlação significativa é o outono (Tabela 2). Isso provavelmente deve-se ao índice utilizar em seu cálculo o Atlântico Tropical, que tem influência 
considerável durante esta estação; ainda assim, o coeficiente de correlação tem valor baixo pois não se verifica o estabelecimento de um dipolo de anomalias de TSM (Fig. 3b). De forma geral, o Atlântico subtropical apresenta uma grande área de anomalias positivas de TSM (Figs. 2b,3b,4b,5b), sugerindo que 0 aquecimento radiativo decorrente da condição de tempo estável está influenciando a TSM do Atlântico, e não o contrário.

$O$ índice da AAO foi incluído nesta análise por possuir conhecida relação com a precipitação no sudeste da América do Sul (Silvestri e Vera, 2003; Rosso et al., 2018; Oliva e Justi da Silva, 2020). A correlação dos eventos de seca com este índice foi notável durante o outono e inverno, embora um sinal claro da AAO não tenha sido percebido nas composições de anomalias de geopotencial da seção anterior (possivelmente o sinal foi enfraquecido pela escala temporal sazonal da análise, pois a variabilidade espacial das anomalias de chuva em escala mensal relacionada à AAO é muito grande - Vasconcellos et al., 2019).

Os sinais da influência da AAO sobre a precipitação durante o inverno em São Paulo são pouco claros (Reboita et al., 2009). Porém, Vasconcellos et al. (2019) documentam uma tendência consistente de anomalias quentes sobre a ESP durante a fase negativa da oscilação nos meses de março, maio, julho e agosto. O predomínio de temperaturas acima da média durante os meses da estação seca quando a AAO está negativa favorece evapotranspiração mais intensa, tornando o índice SPEI (balanço hídrico) mais negativo. Estes resultados sugerem que os eventos de seca durante a estação seca da ESP podem estar mais relacionados ao aumento da perda evaporativa do que à diminuição das chuvas (que já são escassas durante esta época do ano).

\section{CONCLUSÕES}

Os campos médios associados ao início de eventos de seca meteorológica na ESP foram obtidos a partir da reanálise ERA20C. Os eventos foram identificados através do índice SPEI na escala temporal de um mês (SPEI-1), e apresentaram distribuição sazonal aproximadamente constante ao longo do ano, com cerca de 40 eventos iniciados em cada estação. Além dos campos médios, a correlação entre o SPEI-1 e séries temporais de quatro índices climáticos foram calculadas, para complementar a discussão.

Padrões climatológicos de circulação e anomalias característicos do início de eventos de seca na ESP em cada estação do ano estão sumarizados na Figura 6 e descritos abaixo. 


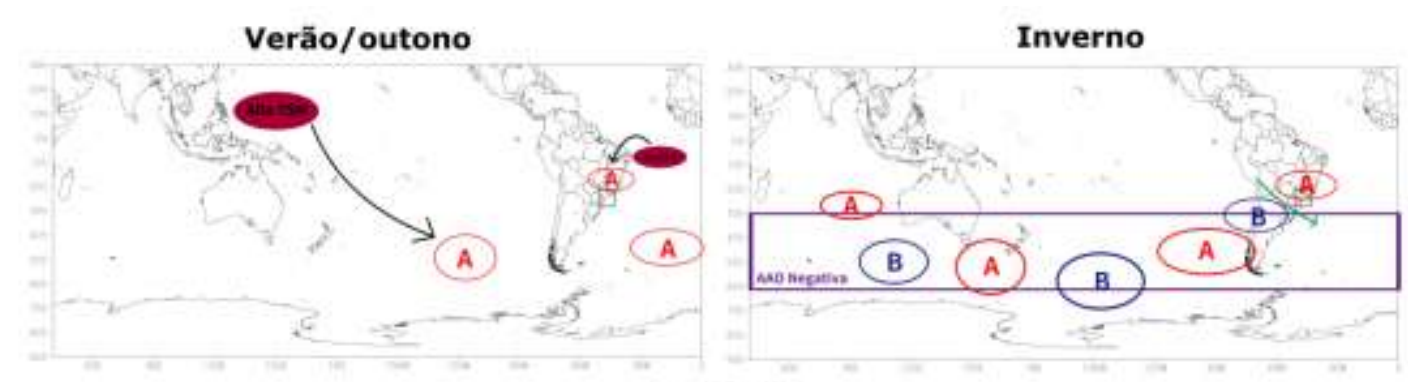

Primavera

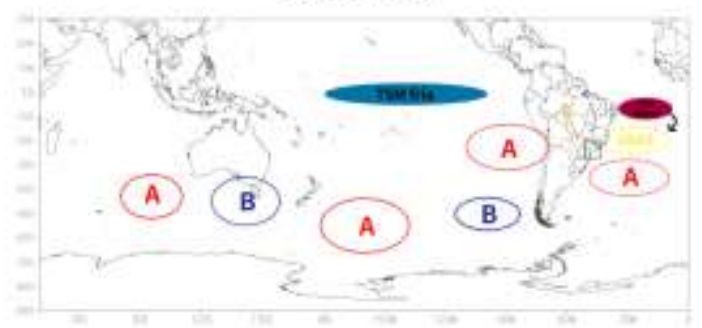

Figura 6 - Esquema das condições climatológicas associadas ao disparo de eventos de seca na ESP (destacada no retângulo), por estação, de acordo com a reanálise ERA-20C para o período 1901-2010. Círculos com as letras " $A$ " e "B" indicam anomalias anticiclônicas e ciclônicas em altos níveis (exceto a anomalia " $A$ " sobre o sudeste no verão/outono, que indica uma alta pressão em superfície induzida por subsidência de compensação). A seta verde no campo de inverno indica a intensificação do jato de altos níveis, e o retângulo roxo representa a fase negativa da AAO. No campo de primavera, o enfraquecimento do jato de baixos níveis é indica pela seta laranja no Brasil central, e o círculo tracejado "ASAS" representa o enfraquecimento da circulação de baixos níveis associada ao ASAS, decorrente da anomalia positiva de TSM.

Durante o verão e o outono, o surgimento de episódios de seca na ESP ocorre associado a uma anomalia quente de TSM a norte/nordeste da Austrália, através da teleconexão atmosférica que posiciona dois anticiclones barotrópicos a oeste e leste da América do Sul. O mecanismo é descrito por Coelho et al. (2016) para a seca de 2014, e parece tratar-se de uma configuração frequente para iniciar secas nestas estações. A manutenção do anticiclone sobre o Pacífico Sul determina um padrão de bloqueio em torno de $120-110^{\circ} \mathrm{W}$, que desfavorece o avanço de perturbações de médios e altos níveis em direção à América do Sul. A desintensificação da ASAS por anomalias quentes de TSM e o enfraquecimento do JBN, associados principalmente no outono ao enfraquecimento do jato subtropical de altos níveis e menor atividade ciclogenética próximo à costa de SP, diminuem o transporte de umidade das regiões tropicais para a ESP. A diminuição de ciclogêneses próximas ao leste de SP concorda com 0 estabelecimento de anomalias positivas de TSM do Atlântico Tropical (Bombardi et al., 2014) e com a fase negativa da AAO (Reboita et al., 2009), conforme mostram as correlações moderadas e significativas obtidas entre o SPEI-1 e o TSA e a AAO no outono. Os eventos de seca iniciados durante o verão não apresentaram relação com os índices analisados neste trabalho, possivelmente porque sua principal forçante é a teleconexão estabelecida com a anomalia de TSM da região da Indonésia; além disso, durante o verão fatores locais podem possuir maior impacto sobre a precipitação de uma região do que a circulação de grande escala.

No inverno, o início dos eventos de seca ocorre associado a um trem de ondas bem definido em altos níveis entre o Oceano Índico e o Atlântico, 
atravessando a bacia do Pacífico. O trem de ondas intensifica o ASAS sobre a costa do Sudeste e o jato de altos níveis sobre o norte da Argentina, favorecendo movimentos descendentes e desviando o transporte de umidade para longe da ESP. Este padrão não é associado às anomalias de TSM do Pacífico Tropical, como mostra a correlação fraca e pouco significativa do SPEI-1 com o índice ONI, e deve estar associado à dinâmica intrassazonal dos extratrópicos (Cazes-Boezio et al., 2003). Além destas anomalias de circulação devido ao trem de ondas, a mais alta correlação foi obtida entre o SPEI-1 do inverno e a AAO. Esta oscilação tem influência mais definida na temperatura do ar do que na precipitação sobre a ESP (Vasconcellos et al., 2019), portanto o início dos eventos de seca meteorológica no inverno nesta região deve ocorrer mais associado ao aumento da evapotranspiração do que à diminuição das chuvas. Tal conclusão sugere melhor desempenho do índice SPEI para identificar eventos secos nesta estação, em relação a outros índices que levam em conta apenas a precipitação.

Nos eventos de seca iniciados na primavera, o escoamento de grande escala remete ao padrão de teleconexão da LN, que enfraquece o jato subtropical sobre o ESP. Em baixos níveis, o JBN e a ASAS enfraquecidos transportam menos umidade em direção a SP e a advecção de ar mais frio pelos ventos anômalos de sul deixam a região mais estável. A importante influência do ENSO sobre a chuva da ESP é reforçada pela concordância dos campos apresentados neste trabalho com a literatura, e pela significativa correlação positiva entre o SPEI-1 e o ONI. A relação entre os eventos de seca desta estação e o índice TSA, indicados pela correlação, serão objeto de estudos posteriores.

Os índices climáticos que apresentaram maior relação com os eventos extremos do balanço hídrico na ESP são a AAO durante o outono e inverno, e o TSA durante o outono e a primavera. O ENSO tem influência apenas na primavera, mas é importante destacar que a presente análise foi realizada apenas para o Niño 3.4, e que outras regiões de anomalias de TSM no Pacífico provocam diferentes respostas sobre a AS (Ashok et al., 2007; Silva e Silva, 2015; Tedeschi et al., 2015). Assim, sugerem-se trabalhos futuros que analisem essas diferentes contribuições.

Anomalias de TSM do Atlântico Subtropical não apresentaram relação com o índice SPEI-1. Embora não pareça ser o principal disparador dos eventos de secas, estudos futuros são necessários para maior compreensão do papel da variabilidade climática associada ao dipolo do Atlântico na região.

\section{AGRADECIMENTOS}

O primeiro e segundo autores agradecem à Fundação de Amparo à Pesquisa do Estado de São Paulo (FAPESP) pelo financiamento desta pesquisa (processo $\mathrm{n}^{\circ}$ 2018/19881-0). Os autores agradecem ao European Centre for Medium-Range Weather Forecasts (ECMWF) pela disponibilização dos dados de reanálise e à colaboração dos revisores anônimos para o aperfeiçoamento deste trabalho. 


\section{REFERÊNCIAS BIBLIOGRÁFICAS}

ANDERSON, W., SEAGER, R., BAETHGEN, W., CANE, M. Life cycles of agriculturally relevant ENSO teleconnections in North and South America. International Journal of Climatology, 37(8), 3297-3318, 2017.

ASHOK, K., BEHERA, S. K., RAO, S. A., WENG, H., YAMAGATA, T. El Niño Modoki and its possible teleconnection. Journal of Geophysical Research: Oceans, 112(C11), 2007.

BALDWIN, M. P. Annular modes in global daily surface pressure. Geophysical Research Letters, 28(21), 4115-4118, 2001.

BOMBARDI, R. J.; CARVALHO, L. M.; JONES, C. REBOITA, M. S. Precipitation over eastern South America and the South Atlantic Sea surface temperature during neutral ENSO periods. Climate Dynamics, 42(5-6), 1553-1568, 2014.

CAMARGO, A.D.; SENTELHAS, P.C. Avaliação do desempenho de diferentes métodos de estimativa da evapotranspiração potencial no Estado de São Paulo, Brasil. Rev. Bras. Agrometeorol., 5, 89-97, 1997.

CARVALHO, L. M. V.; CAVALCANTI, I. F. The South American Monsoon System (SAMS). In: The Monsoons and Climate Change, 121-148. Springer, Cham, 2016.

CAZES-BOEZIO, G.; ROBERTSON, A. W.; MECHOSO, C. R. Seasonal dependence of ENSO teleconnections over South America and relationships with precipitation in Uruguay. Journal of Climate, 16-8, 1159-1176, 2003.

CHEN, T. C., WENG, S. P., SCHUBERT, S. Maintenance of austral summertime upper-tropospheric circulation over tropical South America: The Bolivian highNordeste low system. Journal of the atmospheric sciences, 56(13), 2081-2100., 1999.

CIEMER, C., REHM, L., KURTHS, J., DONNER, R. V., WINKELMANN, R., BOERS, $\mathrm{N}$. An early-warning indicator for Amazon droughts exclusively based on tropical Atlantic sea surface temperatures. Environmental Research Letters, 15(9), 094087, 2020.

COELHO, C. A. D. S., UVO, C. B., AMBRIZZI, T. Exploring the impacts of the tropical Pacific SST on the precipitation patterns over South America during ENSO periods. Theoretical and Applied Climatology, 71(3-4), 185-197, 2002.

COELHO, C. A., DE OliveirA, C. P., AMBRIZZI, T., REBOITA, M. S., CARPENEDO, C. B., CAMPOS, J. L. P. S., ..., DA ROCHA, R. P. The 2014 southeast Brazil austral summer drought: regional scale mechanisms and teleconnections. Climate Dynamics, 46(11-12), 3737-3752, 2016.

DRUMOND, A., STOJANOVIC M., NIETO R., VICENTE-SERRANO S. M., and GIMENO, L. Linking anomalous moisture transport and drought episodes in the IPCC reference regions. Bull. Amer. Meteor. Soc., 100, 1481-1498, 2019.

DUBREUIL, V., FANTE, K. P., PLANCHON, O., SANT'ANNA NETO, J. L. Climate change evidence in Brazil from Köppen's climate annual types frequency. International Journal of Climatology, 39(3), 1446-1456, 2019. 
ENFIELD, D. B., MESTAS-NUÑEZ, A. M., MAYER, D. A., CID-SERRANO, L. How ubiquitous is the dipole relationship in tropical Atlantic sea surface temperatures?. Journal of Geophysical Research: Oceans, 104(C4), 7841-7848, 1999.

FINKE, K., JIMÉNEZ-ESTEVE, B., TASCHETTO, A. S., UMMENHOFER, C. C., BUMKE, K., DOMEISEN, D. I. Revisiting remote drivers of the 2014 drought in South-Eastern Brazil. Climate Dynamics, 1-15, 2020.

FOGT, R. L. et al. Antarctic station-based seasonal pressure reconstructions since 1905: 2. Variability and trends during the twentieth century. Journal of Geophysical Research: Atmospheres, 121, 6, 2836-2856, 2016.

GOZZO, L. F., DA ROCHA, R. P., GIMENO, L., DRUMOND, A. Climatology and numerical case study of moisture sources associated with subtropical cyclogenesis over the southwestern Atlantic Ocean. Journal of Geophysical Research: Atmospheres, 122-11, 5636-5653, 2017.

GOZZO, L. F., PALMA, D. S., CUSTODIO, M. S., MACHADO, J. P. Climatology and Trend of Severe Drought Events in the State of Sao Paulo, Brazil, during the 20th Century. Atmosphere, 10(4), 190, 2019a.

GOZZO, L. F., DRUMOND, A., PALMA, D. S., CUSTODIO, M. D. S. Climatology of Meteorological Drought Events in Sao Paulo (Brazil) and its Representation in ERA5 Reanalysis. AGUFM, 2019, H21K-1895, 2019b.

GRIMM, A. M.; FERRAZ, S. ET; GOMES, J. Precipitation anomalies in southern Brazil associated with El Niño and La Niña events. Journal of climate, v. 11-11, 2863-2880, 1998.

GRIMM, A. M.; BARROS, V. R.; DOYLE, Moira E. Climate variability in southern South America associated with El Niño and La Niña events. Journal of climate, 13-1, 35-58, 2000.

GRIMM, A. M. How do La Niña events disturb the summer monsoon system in Brazil?. Climate Dynamics, 22-2-3, 123-138, 2004.

HARRIS, I.C., JONES, P.D. University of East Anglia Climatic Research Unit, CRU TS4. 01: Climatic Research Unit (CRU) Time-Series (TS) Version 4.01 of HighResolution Gridded Data of Month-by-Month Variation in Climate (Jan.1901-Dec. 2016); Centre for Environmental Data Analysis: Oxfordshire, UK, Volume 4, 2017.

HANEL, M., RAKOVEC, O., MARKONIS, Y., MÁCA, P., SAMANIEGO, L., KYSELÝ, J., KUMAR, R. Revisiting the recent European droughts from a long-term perspective. Scientific reports, 8(1), 1-11, 2018.

JIMÉNEZ-MUÑOZ, J. C., MARENGO, J. A., ALVES, L. M., SULCA, J. C., TAKAHASHI, K., FERRETT, S., \& COLLINS, M. The role of ENSO flavours and TNA on recent droughts over Amazon forests and the Northeast Brazil region. International Journal of Climatology, 2019.

KELLER FILHO, T.; ASSAD, E.D.; LIMA, P.R. Rainfall homogeneous areas in Brazil. Pesqui. Agrop. Bras. 40, 311-322, 2005.

LAU, K.-M., ZHOU, J. Anomalies of the South American summer monsoon associated with the 1997-99 El Nino-Southern Oscillation. International Journal 
of Climatology: A Journal of the Royal Meteorological Society, 23-5, 529-539, 2003.

LIEBMANN, B., MECHOSO, C. R. The south American monsoon system. In: The Global Monsoon System: Research and Forecast, 137-157, 2011.

MARENGO, J. A., SOARES, W. R., SAULO, C., NICOLINI, M. Climatology of the low-level jet east of the Andes as derived from the NCEP-NCAR reanalyses: Characteristics and temporal variability. Journal of climate, 17-12, 2261-2280, 2004.

MARENGO, J. A., TORRES, R. R., ALVES, L. M. Drought in Northeast Brazilpast, present, and future. Theoretical and Applied Climatology, 129(3-4), 1189$1200,2017$.

MCKEE, T.B., DOESKEN, N.J., KLEIST, J. The relationship of drought frequency and duration to time scale. In: Proceedings of the Eighth Conference on Applied Climatology, Anaheim, California,17-22 January 1993. Boston, American Meteorological Society, 179-184, 1993.

MO, K. C., PAEGLE, J. N. The Pacific-South American modes and their downstream effects. International Journal of Climatology: A Journal of the Royal Meteorological Society, 21-10, 1211-1229, 2001.

MORIOKA, Y., TOZUKA, T. e YAMAGATA, T. On the growth and decay of the subtropical dipole mode in the South Atlantic. J. Climate, 24, 5538 - 5554, 2011

MOURA, A. D.,, SHUKLA, J. On the dynamics of droughts in northeast Brazil: Observations, theory and numerical experiments with a general circulation model. Journal of the atmospheric sciences, 38(12), 2653-2675, 1981.

NOBRE, C., MARENGO, J., SELUCHI, M., CUARTAS, L. e ALVES, L. Some Characteristics and Impacts of the Drought and Water Crisis in Southeastern Brazil during 2014 and 2015. Journal of Water Resource and Protection, 8, 252262, 2016.

OLIVA, F. G., JUSTI DA SILVA, M. G. A. Does Antarctic Oscillation have links and influence precipitation over the Uruguay River Drainage Basin in Southeastern South America? Journal of Water and Climate Change, 2020.

PAMPUCH, L. A., DRUMOND, A., GIMENO, L., AMBRIZZI, T. Anomalous patterns of SST and moisture sources in the South Atlantic Ocean associated with dry events in southeastern Brazil. International Journal of Climatology, 36(15), 4913-4928, 2016. .

PENALBA, O. C., RIVERA, J. A. Precipitation response to El Niño/La Niña events in Southern South America-emphasis in regional drought occurrences. Advances in Geosciences, 42, 2016.

PEREIRA, H. R.; REBOITA, M. S.; AMBRIZZI, T. Características da atmosfera na primavera austral durante o El Niño de 2015/2016. Revista Brasileira de Meteorologia, 32-2, 293-310, 2017.

PEReirA, V. R., BLAin, G. C., AVILA, A. M. H. D., PIRES, R. C. D. M., PINTO, H. S. Impacts of climate change on drought: changes to drier conditions at the beginning of the crop growing season in southern Brazil. Bragantia, 77(1), 201$211,2018$. 
POLI, P., HERSBACH, H., DEE, D.P., BERRISFORD, P., SIMMONS, A.J., VITART, F., TRÉMOLET, Y. ERA-20C: An atmospheric reanalysis of the twentieth century. J. Clim., 29, 4083-4097, 2016.

REBOITA, M. S.; AMBRIZZI, T.; ROCHA, R. P. da. Relationship between the southern annular mode and southern hemisphere atmospheric systems. Revista Brasileira de Meteorologia, 24-1, 48-55, 2009.

REBOITA, M. S., DA ROCHA, R. P., AMBRIZZI, T. ,CAETANO, E. An assessment of the latent and sensible heat flux on the simulated regional climate over Southwestern South Atlantic Ocean. Climate dynamics, 34(6), 873-889, 2010a.

REBOITA, M. S., DA ROCHA, R. P., AMBRIZZI, T., \& SUGAHARA, S. South Atlantic Ocean cyclogenesis climatology simulated by regional climate model (RegCM3). Climate Dynamics, 35(7-8), 1331-1347, $2010 \mathrm{~b}$.

REBOITA, M. S., DA ROCHA, R. P., AMBRIZZI, T., GOUVEIA, C. D. Trend and teleconnection patterns in the climatology of extratropical cyclones over the Southern Hemisphere. Climate Dynamics, 45(7-8), 1929-1944, 2015.

REDMOND, K. T. The depiction of drought: A commentary. Bulletin of the American Meteorological Society, 83(8), 1143-1148, 2002.

ROSSO, F. V., BOIASKI, N. T., FERRAZ, S. E. T., ROBLES, T. C. Influence of the Antarctic Oscillation on the South Atlantic Convergence Zone. Atmosphere, 9(11), 431, 2018.

SARAVANAN, R., CHANG, P. Interaction between tropical Atlantic variability and El Nino-Southern oscillation. Journal of climate, 13(13), 2177-2194, 2000.

SILVA, F. D. D. S., PEREIRA-FILHO, A. J., HALLAK, R. Classificação de sistemas meteorológicos e comparação da precipitação estimada pelo radar e medida pela rede telemétrica na Bacia Hidrográfica do Alto Tietê. Revista Brasileira de Meteorologia, 24(3), 292-307, 2009.

SILVA, E. R. L. D. G. e SILVA, M. E. S. Memória de eventos enos na precipitação da América do Sul. Revista do Departamento de Geografia, 30, 34-55, 2015.

SILVA, J. P. R., REBOITA, M. S., ESCOBAR, G. C. J. Caracterização da Zona de Convergência do Atlântico Sul em Campos Atmosféricos recentes. Revista Brasileira de Climatologia, 25, 2019.

SILVESTRI, G. E.; VERA, C. S. Antarctic Oscillation signal on precipitation anomalies over southeastern South America. Geophysical Research Letters, 3021, 2003.

SUN, X., COOK K. H., VIZY E. K. The South Atlantic Subtropical High: Climatology and Interannual Variability. J. Climate, 30, 3279-3296, 2017.

TEDESCHI, R. G.; GRIMM, A. M.; CAVALCANTI, I. F. A. Influence of Central and East ENSO on extreme events of precipitation in South America during austral spring and summer. International Journal of Climatology, 35-8, 2045-2064, 2015.

THORNTHWAITE, C. W. An approach toward a rational classification of climate. Geographical review, 38-1, 55-94, 1948.

TRENBERTH, K. E. The definition of el nino. Bulletin of the American Meteorological Society, 78(12), 2771-2778, 1997. 
UVO, C. B., REPELLI, C. A., ZEBIAK, S. E., KUSHNIR, Y. The relationships between tropical Pacific and Atlantic SST and northeast Brazil monthly precipitation. Journal of Climate, 11(4), 551-562, 1998.

VARGAS, F. F., VEIGA, J. A. P. Padrões atmosféricos e oceânicos associados a eventos secos na bacia amazônica nos anos de 1982/83, 2004/05 e 2009/10. Ciência e Natura, 39(2), 423-435, 2017.

VASCONCELLOS, F. C.; PIZZOCHERO, R. M.; CAVALCANTI, I. F. A. IMPACTO MÊS A MÊS DO MODO ANULAR SUL NA AMÉRICA DO SUL. Anuário do Instituto de Geociências, 42-1, 783-792, 2019.

VEMADO, F., PEREIRA-FILHO, A. J. Severe weather caused by heat island and sea breeze effects in the metropolitan area of São Paulo, Brazil. Advances in Meteorology, 2016.

VICENTE-SERRANO, S. M., BEGUERÍA, S., LÓPEZ-MORENO, J. I. A multiscalar drought index sensitive to global warming: the standardized precipitation evapotranspiration index. Journal of climate, 23(7), 1696-1718, 2010.

WILHITE, D. A., e GLANTZ, M. H. Understanding: the drought phenomenon: the role of definitions. Water international, 10(3), 111-120, 1985.

ZHOU, J, LAU, K. M. Does a monsoon climate exist over South America?. Journal of climate, 11-5, 1020-1040, 1998.

ZHOU, J.; LAU, K.-M. Principal modes of interannual and decadal variability of summer rainfall over South America. International Journal of Climatology: A Journal of the Royal Meteorological Society, 21-13, 1623-1644, 2001. 